\title{
Faddeev-Yakubovsky Technique for Weakly Bound Systems
}

\section{R. Hadizadeh*}

Instituto de Física Teórica, Universidade Estadual Paulista, 01140-070, São Paulo, SP, Brazil E-mail: hadizadedift.unesp.br

\section{T. Yamashita}

Instituto de Física Teórica, Universidade Estadual Paulista, 01140-070, São Paulo, SP, Brazil E-mail: vamashitadift.unesp.br

\section{Lauro Tomio}

Instituto de Física Teórica, Universidade Estadual Paulista, 01140-070, São Paulo, SP, Brazil Instituto de Física, Universidade Federal Fluminense, 24210-346, Niterói, RJ, Brazil

E-mail: tomiodift.unesp.br

\section{A. Delfino}

Instituto de Física, Universidade Federal Fluminense, 24210-346, Niterói, RJ, Brazil

E-mail: delfinodif.uff.der

\section{T. Frederico}

Instituto Tecnológico de Aeronáutica, DCTA, 12228-900, São José dos Campos, SP, Brazil

E-mail: Eobiasdita.br

The Faddeev-Yakubovsky formalism for the study of three- and four-boson bound states have been derived in a partial wave representation for separable potentials and by considering only the s-wave channel contribution. In order to be able to study the weakly three- and four-boson bound states the obtained formalism is simplified for a zero range interaction and the integral equations are regularized by a subtraction technique.

XXXIV edition of the Brazilian Workshop on Nuclear Physics,

5-10 June 2011

Foz do Iguaçu, Parana state, Brasil

\footnotetext{
*Speaker.
} 


\section{Introduction}

Nature shows the existence of weakly bound systems in different sectors, ranging from atomic to nuclear physics. Few-body systems with large scattering length exhibit universal features, which are independent of the details of the interaction, and thus are common to nuclear and atomic systems. Very different methods are used to study the properties of few-body systems, from FaddeevYakubovsky method [U]-[B] to diagonalization methods that rely on an expansion of the wave functions in a complete basis set, like e.g. hyperspherical harmonics and no core shell model. In this paper we present the Faddeev-Yakubovsky formalism to study the three- and four-boson bound states in momentum space. To show the efficiency and accuracy of the method, we have recently investigated the three- and four-boson weakly bound states in the unitary limit (for zero two-body binding) and we have obtained a pretty complete picture of universal tetramers.

\section{Faddeev-Yakubovsky Equations for Four-Boson Bound State}

In this section, we describe the Faddeev-Yakubovsky (FY) formalism for the bound state of four identical bosons. As shown in Eq. (R.T), in a 4B system (with particles called $i, j, k$ and $l$ ) there are 18 different coordinate systems, each associated with a specific two-body partition. These chains have basically two different structures, $3+1$ structure (i.e., a chain in which the four-body system is subdivided into a three-body subsystem and a single particle) and $2+2$ (i.e., subdivision of a four-body system into two two-body subsystems). Clearly, as we know from the Faddeev formalism, each three-body subsystem can also be devided into three $2+1$ chains.

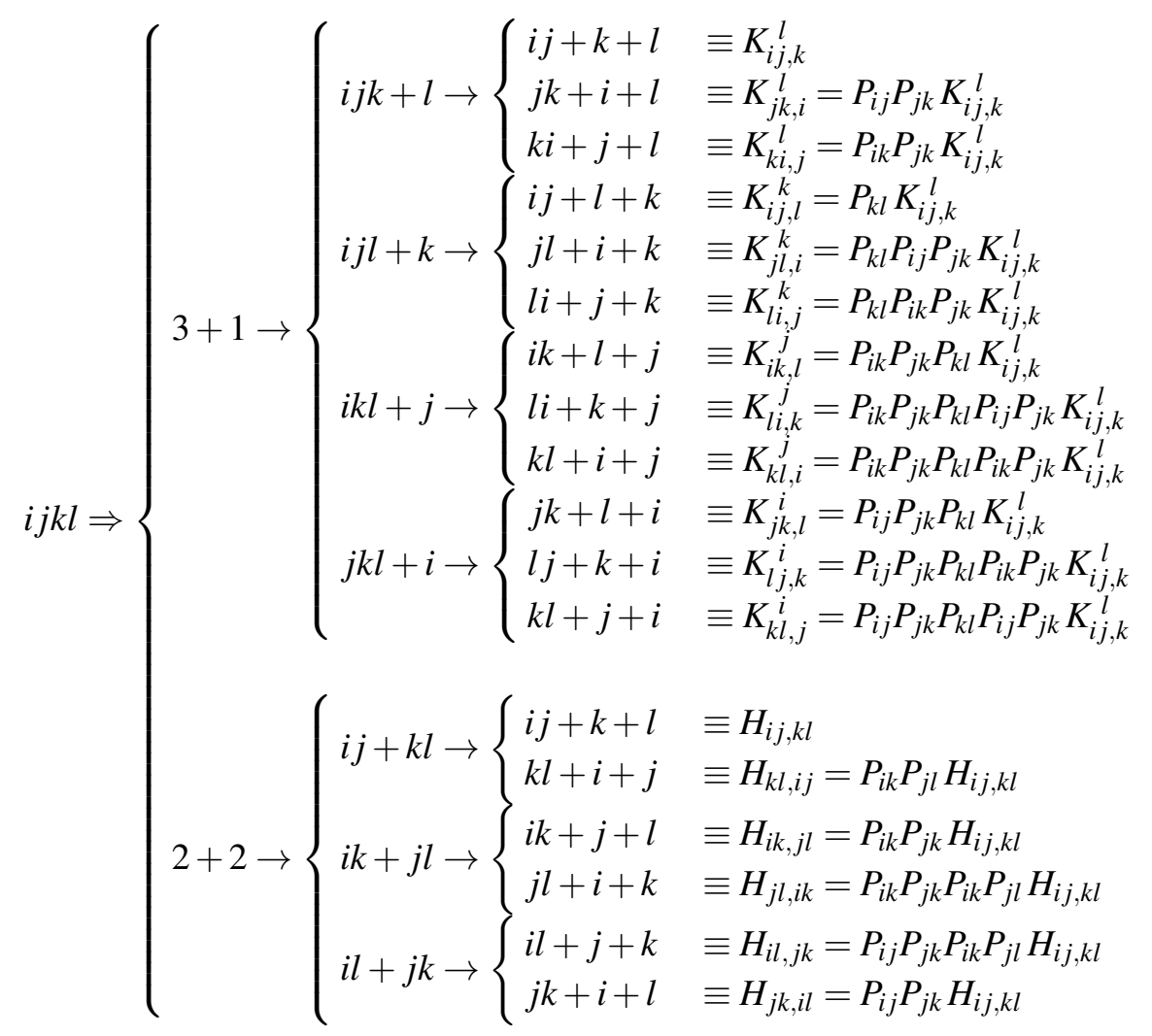


In the following we derive the FY equations. The bound state of four identical particles which interact via pairwise forces $V_{i j}(i j \equiv i j, i k, i l, j k, j l$ and $k l)$ is given by the Schrödinger equation which reads in integral form [四, []]:

$$
|\Psi\rangle=G_{0} \sum_{i<j} V_{i j}|\Psi\rangle
$$

Here the free four-body propagator is given by $G_{0}=\left(E-H_{0}\right)^{-1}$, and $H_{0}$ stands for the free Hamiltonian. Introducing Yakubovsky components $|\Psi\rangle=\sum\left|\psi_{i j}\right\rangle$, with $\left|\psi_{i j}\right\rangle=G_{0} V_{i j}|\Psi\rangle$ leads to the six coupled integral equations:

$$
\left|\psi_{i j}\right\rangle=G_{0} t_{i j} \sum_{k l \neq i j}\left|\psi_{k l}\right\rangle
$$

The operator $t_{i j}$ describes the two-body $t$-matrix in the two-body subsystem $i j$. We can rewrite Eq. (2.3]) as:

$$
\left|\psi_{i j}\right\rangle=G_{0} t_{i j}\left(\left|\psi_{i k}\right\rangle+\left|\psi_{i l}\right\rangle+\left|\psi_{j k}\right\rangle+\left|\psi_{j l}\right\rangle+\left|\psi_{k l}\right\rangle\right)
$$

Among various possibilities to decompose $\left|\psi_{i j}\right\rangle$ into three FY components we choose the following one:

$$
\begin{aligned}
\left|K_{i j, k}^{l}\right\rangle & =G_{0} t_{i j}\left(\left|\psi_{i k}\right\rangle+\left|\psi_{j k}\right\rangle\right) \\
\left|K_{i j, l}^{k}\right\rangle & =G_{0} t_{i j}\left(\left|\psi_{i l}\right\rangle+\left|\psi_{j l}\right\rangle\right) \\
\left|H_{i j, k l}\right\rangle & =G_{0} t_{i j}\left|\psi_{k l}\right\rangle
\end{aligned}
$$

The FY component $\left|K_{i j, k}^{l}\right\rangle\left(\left|H_{i j, k l}\right\rangle\right)$ belongs to a $3+1(2+2)$ partition. They fulfill the following relation:

$$
\left|\psi_{i j}\right\rangle=\left|K_{i j, k}^{l}\right\rangle+\left|K_{i j, l}^{k}\right\rangle+\left|H_{i j, k l}\right\rangle
$$

It is easily seen that every $\left|\psi_{i j}\right\rangle$ component contains two $3+1$ type chains and one $2+2$ type chain, therefore the total wave function $|\Psi\rangle$ contains twelve different $3+1$ type chains and six $2+2$ type chains. So altogether one has eighteen FY components. If we consider identical particles (here bosons, since we are omitting spin), the four-body wave function $|\Psi\rangle$ has to be totally symmetric. As a consequence all twelve components of $3+1$ type are identical in their functional form and only the particles are permuted. The same is true for the six components of $2+2$ type. Thus it is sufficient to consider only two independent FY components corresponding to the $3+1$ and $2+2$ partitions,

$$
\begin{array}{r}
|K\rangle=\left|K_{i j, k}^{l}\right\rangle \\
|H\rangle=\left|H_{i j, k l}\right\rangle
\end{array}
$$

After the straightforward derivation the 18 coupled FY components shrink to two coupled FY equations:

$$
\begin{array}{r}
\left|K_{i j, k}^{l}\right\rangle=G_{0} t_{i j} P\left[\left(1+P_{k l}\right)\left|K_{i j, k}^{l}\right\rangle+\left|H_{i j, k l}\right\rangle\right] \\
\left|H_{i j, k l}\right\rangle=G_{0} t_{i j} \tilde{P}\left[\left(1+P_{k l}\right)\left|K_{i j, k}^{l}\right\rangle+\left|H_{i j, k l}\right\rangle\right]
\end{array}
$$


where $P_{i j}$ is the permutation operator between the $i-t h$ and $j-t h$ particle, and

$$
\begin{aligned}
& P=P_{i j} P_{j k}+P_{i k} P_{j k} \\
& \tilde{P}=P_{i k} P_{j l}
\end{aligned}
$$

The total four-body wave function is then given as:

$$
\begin{aligned}
|\Psi\rangle & =\left(1+P+P_{k l} P+\tilde{P}\right)\left[\left(1+P_{k l}\right)\left|K_{i j, k}^{l}\right\rangle+\left|H_{i j, k l}\right\rangle\right] \\
& =\left(1+(1+P) P_{k l}\right)(1+P)\left|K_{i j, k}^{l}\right\rangle+(1+P)(1+\tilde{P})\left|H_{i j, k l}\right\rangle
\end{aligned}
$$

The symmetry property of $|K\rangle$ under exchange of particles $i$ and $j$, and $|H\rangle$ under separate exchanges of particles $i, j$ and $k, l$ guarantee that $|\Psi\rangle$ is totally symmetric.

\section{Representation of Faddeev-Yakubovsky Equations in Momentum Space}

In this section for simplification of notation, we use the labels 1,2,3 and 4 for particles. So, we can rewrite Eq. (2.8) as:

$$
\begin{aligned}
& |K\rangle=G_{0} t_{12} P\left[\left(1+P_{34}\right)|K\rangle+|H\rangle\right] \\
& |H\rangle=G_{0} t_{12} \tilde{P}\left[\left(1+P_{34}\right)|K\rangle+|H\rangle\right]
\end{aligned}
$$

In order to solve the coupled Eqs. (B. D) in momentum space one should project them into standard sets of Jacobi momenta, corresponding to both $3+1\left(\left|\mathbf{u}_{1} \mathbf{u}_{2} \mathbf{u}_{3}\right\rangle\right)$ and $2+2\left(\left|\mathbf{v}_{1} \mathbf{v}_{2} \mathbf{v}_{3}\right\rangle\right)$ chains. The standard Jacobi momenta for 4B system are defined as:

$$
\begin{aligned}
& \left\{\begin{array}{l}
\mathbf{u}_{1}=\frac{1}{2}\left(\mathbf{k}_{1}-\mathbf{k}_{2}\right) \\
\mathbf{u}_{2}=\frac{2}{3}\left(\mathbf{k}_{3}-\left(\mathbf{k}_{1}+\mathbf{k}_{2}\right)\right) \\
\mathbf{u}_{3}=\frac{3}{4}\left(\mathbf{k}_{4}-\frac{1}{3}\left(\mathbf{k}_{1}+\mathbf{k}_{2}+\mathbf{k}_{3}\right)\right)
\end{array}\right. \\
& \left\{\begin{array}{l}
\mathbf{v}_{1}=\frac{1}{2}\left(\mathbf{k}_{1}-\mathbf{k}_{2}\right) \\
\mathbf{v}_{2}=\frac{1}{2}\left(\mathbf{k}_{1}+\mathbf{k}_{2}\right)-\frac{1}{2}\left(\mathbf{k}_{3}+\mathbf{k}_{4}\right) \\
\mathbf{v}_{3}=\frac{1}{2}\left(\mathbf{k}_{3}-\mathbf{k}_{4}\right)
\end{array}\right.
\end{aligned}
$$

Since we are going to study the weakly $4 \mathrm{~B}$ bound state and we just consider the s-wave channel contribution, we introduce the projection operators corresponding to each Jacobi momenta set in partial wave representation as:

$$
\begin{aligned}
& |u\rangle=\left|u_{1} u_{2} u_{3}\right\rangle \\
& |v\rangle=\left|v_{1} v_{2} v_{3}\right\rangle
\end{aligned}
$$


where the projection operators follow the completeness relation:

$$
\int D^{3} A|A\rangle\langle A|=1
$$

where $A$ indicates each one of $u$ and $v$ vectors and $D^{3} A \equiv A_{1}^{2} d A_{1} A_{2}^{2} d A_{2} A_{3}^{2} d A_{3}$. Clearly the projection operator $|u\rangle$ is adequate to expand FY component $|K\rangle$ and correspondingly the projection operator $|v\rangle$ is adequate for $|H\rangle$. Let us now represent the coupled equations, Eq. (B.D), with respect to the projection operators which have been introduced in Eq. (3.4):

$$
\begin{aligned}
& \langle u \mid K\rangle=\left\langle u\left|G_{0} t P\left(1+P_{34}\right)\right| K\right\rangle+\left\langle u\left|G_{0} t P\right| H\right\rangle \\
& \langle v \mid H\rangle=\left\langle v\left|G_{0} t \tilde{P}\left(1+P_{34}\right)\right| K\right\rangle+\left\langle v\left|G_{0} t \tilde{P}\right| H\right\rangle
\end{aligned}
$$

It is convenient to insert again the completeness relations between permutation operators, it results:

$$
\begin{aligned}
\langle u \mid K\rangle & =\int D^{3} u^{\prime \prime}\left\langle u\left|G_{0} t P\right| u^{\prime \prime}\right\rangle\left\langle u^{\prime \prime} \mid K\right\rangle \\
& +\int D^{3} u^{\prime \prime} \int D^{3} u^{\prime \prime \prime}\left\langle u\left|G_{0} t P\right| u^{\prime \prime}\right\rangle\left\langle u^{\prime \prime}\left|P_{34}\right| u^{\prime \prime \prime}\right\rangle\left\langle u^{\prime \prime \prime} \mid K\right\rangle \\
& +\int D^{3} u^{\prime \prime} \int D^{3} v^{\prime}\left\langle u\left|G_{0} t P\right| u^{\prime \prime}\right\rangle\left\langle u^{\prime \prime} \mid v^{\prime}\right\rangle\left\langle v^{\prime} \mid H\right\rangle \\
\langle v \mid H\rangle & =\int D^{3} v^{\prime \prime} \int D^{3} u^{\prime}\left\langle v\left|G_{0} t \tilde{P}\right| v^{\prime \prime}\right\rangle\left\langle v^{\prime \prime} \mid u^{\prime}\right\rangle\left\langle u^{\prime} \mid K\right\rangle \\
& +\int D^{3} v^{\prime \prime} \int D^{3} u^{\prime} \int D^{3} u^{\prime \prime}\left\langle v\left|G_{0} t \tilde{P}\right| v^{\prime \prime}\right\rangle\left\langle v^{\prime \prime} \mid u^{\prime}\right\rangle\left\langle u^{\prime}\left|P_{34}\right| u^{\prime \prime}\right\rangle\left\langle u^{\prime \prime} \mid K\right\rangle \\
& +\int D^{3} v^{\prime \prime}\left\langle v\left|G_{0} t \tilde{P}\right| v^{\prime \prime}\right\rangle\left\langle v^{\prime \prime} \mid H\right\rangle
\end{aligned}
$$

For evaluating the coupled equations, Eq. (B.7), we need to evaluate the following matrix elements:

$$
\begin{aligned}
& \left\langle u\left|G_{0} t P\right| u^{\prime \prime}\right\rangle \\
& \left\langle v\left|G_{0} t \tilde{P}\right| v^{\prime \prime}\right\rangle \\
& \left\langle u^{\prime \prime}\left|P_{34}\right| u^{\prime \prime \prime}\right\rangle, \quad\left\langle u^{\prime}\left|P_{34}\right| u^{\prime \prime}\right\rangle \\
& \left\langle u^{\prime \prime} \mid v^{\prime}\right\rangle, \quad\left\langle v^{\prime \prime} \mid u^{\prime}\right\rangle
\end{aligned}
$$

For evaluating the first term, Eq. (3.8), we should insert again a completeness relation between the two-body $t$-matrix operator and the permutation operator $P$ as:

$$
\left\langle u\left|G_{0} t P\right| u^{\prime \prime}\right\rangle=G_{0}\left(u_{1}, u_{2}, u_{3}\right) \times \int D^{3} u^{\prime}\left\langle u|t| u^{\prime}\right\rangle\left\langle u^{\prime}|P| u^{\prime \prime}\right\rangle
$$

where the matrix elements of the two-body $t$-matrix (for a separable potential) and permutation operator $P$ are evaluated separately as:

$$
\begin{aligned}
\left\langle u|t| u^{\prime}\right\rangle & =\frac{\delta\left(u_{2}^{\prime}-u_{2}\right)}{u_{2}^{\prime 2}} \frac{\delta\left(u_{3}^{\prime}-u_{3}\right)}{u_{2}^{\prime 3}} 4 \pi \chi\left(u_{1}\right) \chi\left(u_{1}^{\prime}\right) \tau(\varepsilon) \\
\varepsilon & =E-\frac{3 u_{2}^{2}}{4 m}-\frac{2 u_{3}^{2}}{3 m}
\end{aligned}
$$




$$
\begin{aligned}
\left\langle u^{\prime}|P| u^{\prime \prime}\right\rangle & =\int d x \frac{\delta\left(u_{1}^{\prime}-\Pi_{1}\left(u_{2}^{\prime}, u_{2}^{\prime \prime}\right)\right)}{u_{1}^{\prime 2}} \frac{\delta\left(u_{1}^{\prime \prime}-\Pi_{1}\left(u_{2}^{\prime \prime}, u_{2}^{\prime}\right)\right)}{u_{1}^{\prime 2}} \frac{\delta\left(u_{3}^{\prime \prime}-u_{3}^{\prime}\right)}{u_{3}^{\prime \prime 2}} \\
\Pi_{1}\left(u_{2}^{\prime}, u_{2}^{\prime \prime}\right) & =\left|\frac{1}{2} \mathbf{u}_{2}^{\prime}+\mathbf{u}_{2}^{\prime \prime}\right|=\sqrt{\frac{1}{4} u_{2}^{\prime 2}+u_{2}^{\prime \prime 2}+u_{2}^{\prime} u_{2}^{\prime 2} x} \\
\Pi_{1}\left(u_{2}^{\prime \prime}, u_{2}^{\prime}\right) & =\left|\frac{1}{2} \mathbf{u}_{2}^{\prime \prime}+\mathbf{u}_{2}^{\prime}\right|=\sqrt{\frac{1}{4} u_{2}^{\prime \prime 2}+u_{2}^{\prime 2}+u_{2}^{\prime} u_{2}^{\prime \prime 2} x}
\end{aligned}
$$

For evaluation the matrix elements of permutation operator $P$ we have used the relation between Jacobi momenta in different two-body subsystems $(312,4),(231,4)$ and $(123,4)$. Inserting Eqs. (B.13) and (B..4) into Eq. (B.J2) leads to:

$$
\begin{aligned}
\left\langle u\left|G_{0} t P\right| u^{\prime \prime}\right\rangle & =4 \pi G_{0}\left(u_{1}, u_{2}, u_{3}\right) \chi\left(u_{1}\right) \tau(\varepsilon) \\
& * \int d x \chi\left(\Pi_{1}\left(u_{2}, u_{2}^{\prime \prime}\right)\right) \frac{\delta\left(u_{1}^{\prime \prime}-\Pi_{1}\left(u_{2}^{\prime \prime}, u_{2}\right)\right)}{u_{1}^{\prime 2}} \frac{\delta\left(u_{3}^{\prime \prime}-u_{3}\right)}{u_{3}^{\prime \prime 2}}
\end{aligned}
$$

Representation of the second term, Eq. (B.9), follows the similar steps:

$$
\left\langle v\left|G_{0} t \tilde{P}\right| v^{\prime \prime}\right\rangle=G_{0}\left(v_{1}, v_{2}, v_{3}\right) \int D^{3} v^{\prime}\left\langle v|t| v^{\prime}\right\rangle\left\langle v^{\prime}|\tilde{P}| v^{\prime \prime}\right\rangle
$$

The matrix elements of the two-body $t$-matrix and the permutation operator $\tilde{P}$ are evaluated as:

$$
\begin{gathered}
\left\langle v|t| v^{\prime \prime}\right\rangle=\frac{\delta\left(v_{2}^{\prime}-v_{2}\right)}{v_{2}^{\prime 2}} \frac{\delta\left(v_{3}^{\prime}-v_{3}\right)}{v_{2}^{\prime 3}} 4 \pi \chi\left(v_{1}\right) \chi\left(v_{1}^{\prime}\right) \tau\left(\varepsilon^{*}\right) \\
\varepsilon^{*}=E-\frac{v_{2}^{2}}{2 m}-\frac{v_{3}^{2}}{m} \\
\left\langle v^{\prime}|\tilde{P}| v^{\prime \prime}\right\rangle=\frac{\delta\left(v_{1}^{\prime}-v_{3}^{\prime \prime}\right)}{v_{1}^{\prime 2}} \frac{\delta\left(v_{3}^{\prime}-v_{1}^{\prime \prime}\right)}{v_{3}^{\prime 2}} \frac{\delta\left(v_{2}^{\prime}-v_{2}^{\prime \prime}\right)}{v_{2}^{\prime 2}}
\end{gathered}
$$

Inserting Eqs. (B.TC) and (B.J8) into Eq. (B.T6) leads to:

$$
\left\langle v\left|G_{0} t \tilde{P}\right| v^{\prime \prime}\right\rangle=4 \pi G_{0}\left(v_{1}, v_{2}, v_{3}\right) \chi\left(v_{1}\right) \chi\left(v_{3}^{\prime \prime}\right) \tau\left(\varepsilon^{*}\right) \frac{\delta\left(v_{3}-v_{1}^{\prime \prime}\right)}{v_{3}^{2}} \frac{\delta\left(v_{2}-v_{2}^{\prime \prime}\right)}{v_{2}^{2}}
$$

For the evaluation of the third term, Eq. (B.10), we should use the relation between Jacobi momenta in different chains $(123,4)$ and $(124,3)$, which leads to:

$$
\begin{aligned}
\left\langle u^{\prime \prime}\left|P_{34}\right| u^{\prime \prime \prime}\right\rangle & =\frac{\delta\left(u_{1}^{\prime \prime}-u_{1}^{\prime \prime \prime}\right)}{u_{1}^{\prime \prime \prime}} \frac{1}{2} \int d x^{\prime} \frac{\delta\left(u_{2}^{\prime \prime \prime}-\Pi_{2}\left(u_{2}^{\prime \prime}, u_{3}^{\prime \prime}, x^{\prime}\right)\right)}{u_{2}^{\prime \prime \prime 2}} \frac{\delta\left(u_{3}^{\prime \prime \prime}-\Pi_{3}\left(u_{2}^{\prime \prime}, u_{3}^{\prime \prime}, x^{\prime}\right)\right)}{u_{3}^{\prime \prime \prime 2}} \\
\Pi_{2}\left(u_{2}^{\prime \prime}, u_{3}^{\prime \prime}, x^{\prime}\right) & =\left|\frac{1}{3} \mathbf{u}_{2}^{\prime \prime}+\frac{8}{9} \mathbf{u}_{3}^{\prime \prime}\right|=\sqrt{\frac{1}{9} u_{2}^{\prime \prime 2}+\frac{64}{81} u_{3}^{\prime \prime 2}+\frac{16}{27} u_{2}^{\prime \prime} u_{3}^{\prime \prime} x^{\prime}} \\
\Pi_{3}\left(u_{2}^{\prime \prime}, u_{3}^{\prime \prime}, x^{\prime}\right) & =\left|\mathbf{u}_{2}^{\prime \prime}-\frac{1}{3} \mathbf{u}_{3}^{\prime \prime}\right|=\sqrt{u_{2}^{\prime \prime 2}+\frac{1}{9} u_{3}^{\prime \prime 2}-\frac{2}{3} u_{2}^{\prime \prime} u_{3}^{\prime \prime} x^{\prime}}
\end{aligned}
$$


And finally for evaluation the fourth terms, Eq. (B.D), we should use the relation between Jacobi momenta in two naturally different chains $(123,4)$ and $(12,34)$, which leads to:

$$
\begin{aligned}
\left\langle u^{\prime \prime} \mid v^{\prime}\right\rangle & =\frac{\delta\left(v_{1}^{\prime}-u_{1}^{\prime \prime}\right)}{v_{1}^{\prime 2}} \frac{1}{2} \int d x^{\prime} \frac{\delta\left(v_{3}^{\prime}-\Pi_{4}\left(u_{2}^{\prime \prime}, u_{3}^{\prime \prime}, x^{\prime}\right)\right)}{v_{3}^{\prime 2}} \frac{\delta\left(v_{2}^{\prime}-\Pi_{5}\left(u_{2}^{\prime \prime}, u_{3}^{\prime \prime}, x^{\prime}\right)\right)}{v_{2}^{\prime 2}} \\
\Pi_{4}\left(u_{2}^{\prime \prime}, u_{3}^{\prime \prime}, x^{\prime}\right) & =\left|\frac{1}{2} \mathbf{u}_{2}^{\prime \prime}+\frac{2}{3} \mathbf{u}_{3}^{\prime \prime}\right|=\sqrt{\frac{1}{4} u_{2}^{\prime \prime 2}+\frac{4}{9} u_{3}^{\prime \prime 2}+\frac{2}{3} u_{2}^{\prime \prime} u_{3}^{\prime \prime} x^{\prime}} \\
\Pi_{3}\left(u_{2}^{\prime \prime}, u_{3}^{\prime \prime}, x^{\prime}\right) & =\left|\mathbf{u}_{2}^{\prime \prime}-\frac{2}{3} \mathbf{u}_{3}^{\prime \prime}\right|=\sqrt{u_{2}^{\prime \prime 2}+\frac{4}{9} u_{3}^{\prime \prime 2}-\frac{4}{3} u_{2}^{\prime \prime} u_{3}^{\prime \prime} x^{\prime}} \\
\left\langle v^{\prime \prime} \mid u^{\prime}\right\rangle & =\frac{\delta\left(u_{1}^{\prime}-v_{1}^{\prime \prime}\right)}{u_{1}^{\prime 2}} \frac{1}{2} \int d x \frac{\delta\left(u_{2}^{\prime}-\Pi_{6}\left(v_{2}^{\prime \prime}, v_{3}^{\prime \prime}, x\right)\right.}{u_{2}^{\prime 2}} \frac{\delta\left(u_{3}^{\prime}-\Pi_{7}\left(v_{2}^{\prime \prime}, v_{3}^{\prime \prime}, x\right)\right)}{u_{3}^{\prime 2}} \\
\Pi_{6}\left(v_{2}^{\prime \prime}, v_{3}^{\prime \prime}, x\right) & =\frac{2}{3}\left|\mathbf{v}_{2}^{\prime \prime}+\mathbf{v}_{3}^{\prime \prime}\right|=\frac{2}{3} \sqrt{v_{2}^{\prime \prime 2}+v_{3}^{\prime \prime 2}+2 v_{2}^{\prime \prime} v_{3}^{\prime \prime} x} \\
\Pi_{7}\left(v_{2}^{\prime \prime}, v_{3}^{\prime \prime}, x\right) & =\left|\mathbf{v}_{3}^{\prime \prime}-\frac{1}{2} \mathbf{v}_{2}^{\prime \prime}\right|=\sqrt{v_{3}^{\prime \prime 2}+\frac{1}{4} v_{2}^{\prime \prime 2}-v_{2}^{\prime \prime} v_{3}^{\prime \prime} x}
\end{aligned}
$$

Finally inserting Eqs. (B..5), (B., $)$, (B.20) and (B.22) in Eq. (B.7) yields:

$$
\begin{aligned}
K\left(u_{1}, u_{2}, u_{3}\right) & =4 \pi G_{0}\left(u_{1}, u_{2}, u_{3}\right) \chi\left(u_{1}\right) \tau(\varepsilon) \\
& *\left[\int d u_{2}^{\prime} u_{2}^{\prime 2} \int d x \chi\left(\Pi\left(u_{2}, u_{2}^{\prime}\right)\right) K\left(\Pi\left(u_{2}^{\prime}, u_{2}\right), u_{2}^{\prime}, u_{3}\right)\right. \\
& +\frac{1}{2} \int d u_{2}^{\prime} u_{2}^{\prime 2} \int d x \int d x^{\prime} \chi\left(\Pi\left(u_{2}, u_{2}^{\prime}\right)\right) K\left(\Pi\left(u_{2}^{\prime}, u_{2}\right), \Pi_{2}\left(u_{2}^{\prime}, u_{3}, x^{\prime}\right), \Pi_{3}\left(u_{2}^{\prime}, u_{3}, x^{\prime}\right)\right) \\
& \left.+\frac{1}{2} \int d u_{2}^{\prime} u_{2}^{\prime 2} \int d x \int d x^{\prime} \chi\left(\Pi\left(u_{2}, u_{2}^{\prime}\right)\right) H\left(\Pi\left(u_{2}^{\prime}, u_{2}\right), \Pi_{4}\left(u_{2}^{\prime}, u_{3}, x^{\prime}\right), \Pi_{5}\left(u_{2}^{\prime}, u_{3}, x^{\prime}\right)\right)\right] \\
H\left(v_{1}, v_{2}, v_{3}\right) & =4 \pi G_{0}\left(v_{1}, v_{2}, v_{3}\right) \chi\left(v_{1}\right) \tau\left(\varepsilon^{*}\right) \\
& *\left[\int d v_{3}^{\prime} v_{3}^{\prime 2} \int d x \chi\left(v_{3}^{\prime}\right) K\left(v_{3}, \Pi_{6}\left(v_{2}, v_{3}^{\prime}, x\right), \Pi_{7}\left(v_{2}, v_{3}^{\prime}, x\right)\right)\right. \\
& \left.+\int d v_{3}^{\prime} v_{3}^{\prime 2} \chi\left(v_{3}^{\prime}\right) H\left(v_{3}, v_{2}, v_{3}^{\prime}\right)\right]
\end{aligned}
$$

By considering the following definitions of Yakubovsky components:

$$
\begin{aligned}
K\left(u_{1}, u_{2}, u_{3}\right) & =G_{0}\left(u_{1}, u_{2}, u_{3}\right) \chi\left(u_{1}\right) \mathscr{K}\left(u_{2}, u_{3}\right) \\
H\left(v_{1}, v_{2}, v_{3}\right) & =G_{0}\left(v_{1}, v_{2}, v_{3}\right) \chi\left(v_{1}\right) \mathscr{H}\left(v_{2}, v_{3}\right)
\end{aligned}
$$


the final form of coupled Yakubovsky integral equations can be obtained as:

$$
\begin{aligned}
\mathscr{K}\left(u_{2}, u_{3}\right)= & 4 \pi \tau(\varepsilon) \\
* & {\left[\int d u_{2}^{\prime} u_{2}^{\prime 2} \int d x \chi\left(\Pi\left(u_{2}, u_{2}^{\prime}\right)\right) \chi\left(\Pi\left(u_{2}^{\prime}, u_{2}\right)\right) G_{0}\left(\Pi\left(u_{2}^{\prime}, u_{2}\right), u_{2}^{\prime}, u_{3}\right) \mathscr{K}\left(u_{2}^{\prime}, u_{3}\right)\right.} \\
+ & \frac{1}{2} \int d u_{2}^{\prime} u_{2}^{\prime 2} \int d x \int d x^{\prime} \chi\left(\Pi\left(u_{2}, u_{2}^{\prime}\right)\right) \chi\left(\Pi\left(u_{2}^{\prime}, u_{2}\right)\right) \\
& \times G_{0}\left(\Pi\left(u_{2}^{\prime}, u_{2}\right), \Pi_{2}\left(u_{2}^{\prime}, u_{3}, x^{\prime}\right), \Pi_{3}\left(u_{2}^{\prime}, u_{3}, x^{\prime}\right)\right) \mathscr{K}\left(\Pi_{2}\left(u_{2}^{\prime}, u_{3}, x^{\prime}\right), \Pi_{3}\left(u_{2}^{\prime}, u_{3}, x^{\prime}\right)\right) \\
+ & \frac{1}{2} \int d u_{2}^{\prime} u_{2}^{\prime 2} \int d x \int d x^{\prime} \chi\left(\Pi\left(u_{2}, u_{2}^{\prime}\right)\right) \chi\left(\Pi\left(u_{2}^{\prime}, u_{2}\right)\right) \\
& \left.\times G_{0}\left(\Pi\left(u_{2}^{\prime}, u_{2}\right), \Pi_{4}\left(u_{2}^{\prime}, u_{3}, x^{\prime}\right), \Pi_{5}\left(u_{2}^{\prime}, u_{3}, x^{\prime}\right)\right) \mathscr{H}\left(\Pi_{4}\left(u_{2}^{\prime}, u_{3}, x^{\prime}\right), \Pi_{5}\left(u_{2}^{\prime}, u_{3}, x^{\prime}\right)\right)\right] \\
\mathscr{H}\left(v_{2}, v_{3}\right)= & 4 \pi \tau\left(\varepsilon^{*}\right) \\
* & {\left[\int d v_{3}^{\prime} v_{3}^{\prime 2} \int d x \chi\left(v_{3}^{\prime}\right) \chi\left(v_{3}\right)\right.} \\
& \times G_{0}\left(v_{3}, \Pi_{6}\left(v_{2}, v_{3}^{\prime}, x\right), \Pi_{7}\left(v_{2}, v_{3}^{\prime}, x\right)\right) \mathscr{K}\left(\Pi_{6}\left(v_{2}, v_{3}^{\prime}, x\right), \Pi_{7}\left(v_{2}, v_{3}^{\prime}, x\right)\right) \\
+ & \left.\int d v_{3}^{\prime} v_{3}^{\prime 2} \chi\left(v_{3}^{\prime}\right) \chi\left(v_{3}\right) G_{0}\left(v_{3}, v_{2}, v_{3}^{\prime}\right) \mathscr{H}\left(v_{2}, v_{3}^{\prime}\right)\right]
\end{aligned}
$$

\section{Zero-range interaction}

The momentum space representation of a zero-range interaction $V(\mathbf{r})=(2 \pi)^{3} \lambda \delta(\mathbf{r})$, characterized by the two-body coupling constant $\lambda$ is as:

$$
\left\langle\mathbf{p}|V| \mathbf{p}^{\prime}\right\rangle=\lambda\langle\mathbf{p} \mid \chi\rangle\left\langle\chi \mid \mathbf{p}^{\prime}\right\rangle \quad ; \quad\langle\mathbf{p} \mid \chi\rangle \equiv \chi(\mathbf{p})=\int d^{3} r e^{i \mathbf{p} . \mathbf{r}} \delta(\mathbf{r})=1 .
$$

The two-body $t$-matrix, $t(\varepsilon)=|\chi\rangle \tau(\varepsilon)\langle\chi|$, can be obtained by analytical solution of the inhomogeneous Lippmann-Schwinger equation as:

$$
\tau(\varepsilon)=\left[\lambda^{-1}-\int d^{3} p \frac{1}{\varepsilon-p^{2}}\right]^{-1}
$$

The coupling constant $\lambda$ is fixed by one physical input, for example, the pole position of the two-body scattering amplitude at $B_{2}$;

$$
\lambda^{-1}=\int d^{3} p \frac{1}{B_{2}-p^{2}}
$$

The above value of $\lambda$, when substituted in Eq. (4.2), provides the cancelation of linear divergence of momentum integral and leads to renormalized two-body $t$-matrix as: 


$$
\tau(\varepsilon)=\frac{1}{2 \pi^{2}}\left(\sqrt{-B_{2}}-\sqrt{-\varepsilon}\right)^{-1}=\frac{1}{2 \pi^{2}}\left(\frac{1}{a}-\sqrt{-\varepsilon}\right)^{-1} .
$$

\section{Coupled Yakubovsky Integral Equations for Zero-range interaction}

The coupled integral equations (B.25) can be simplified for a zero range interaction $(\chi(\mathbf{p})=1)$. If we ignore the fourth particle, the coupled Yakubovsky integral equations can be simplified to Faddeev integral equation. The subtraction technique can be used to be able to obtain the converged results for binding energy of trimer from solution of Faddeev integral equation by using a zero range interaction. To this aim one need a three-body parameter, $\mu_{3}$, and the regularization of the momentum integration is done through the substitution of the free three-body Green's function by a subtracted form:

$$
G_{0}^{(3)}=\frac{1}{E-H_{0}}-\frac{1}{-\mu_{3}^{2}-H_{0}}
$$

Now, by adding the fourth particle to the three-body system, one need also to regularize the new terms which appear in presence of fourth particle in coupled Yakubovsky integral equations. To this aim one can introduce a new parameter, i.e. four-body scale $\mu_{4}$, to regularize these new terms and the free four-body Green's function can be substituted by a subtracted form:

$$
G_{0}^{(4)}=\frac{1}{E-H_{0}}-\frac{1}{-\mu_{4}^{2}-H_{0}}
$$

So, for regularization of the first term of coupled integral equation (B.25), which describe the 3B subsystem $(i j k)$, one can use a three-body scale and for other terms, which appear in presence of fourth particle, a new four-body scale can be used. By these considerations, he subtracted form of the Yakubovsky integral equations are given by:

$$
\begin{aligned}
& \mathscr{K}\left(u_{2}, u_{3}\right)=4 \pi \tau(\varepsilon) \int d u_{2}^{\prime} u_{2}^{\prime 2} \int d x \\
& \times\left[G_{0}^{(3)}\left(\Pi\left(u_{2}^{\prime}, u_{2}\right), u_{2}^{\prime}, u_{3}\right) \mathscr{K}\left(u_{2}^{\prime}, u_{3}\right)\right. \\
& \quad+\frac{1}{2} \int d x^{\prime} G_{0}^{(4)}\left(\Pi\left(u_{2}^{\prime}, u_{2}\right), \Pi_{2}\left(u_{2}^{\prime}, u_{3}, x^{\prime}\right), \Pi_{3}\left(u_{2}^{\prime}, u_{3}, x^{\prime}\right)\right) \mathscr{K}\left(\Pi_{2}\left(u_{2}^{\prime}, u_{3}, x^{\prime}\right), \Pi_{3}\left(u_{2}^{\prime}, u_{3}, x^{\prime}\right)\right) \\
& \left.\quad+\frac{1}{2} \int d x^{\prime} G_{0}^{(4)}\left(\Pi\left(u_{2}^{\prime}, u_{2}\right), \Pi_{4}\left(u_{2}^{\prime}, u_{3}, x^{\prime}\right), \Pi_{5}\left(u_{2}^{\prime}, u_{3}, x^{\prime}\right)\right) \mathscr{H}\left(\Pi_{4}\left(u_{2}^{\prime}, u_{3}, x^{\prime}\right), \Pi_{5}\left(u_{2}^{\prime}, u_{3}, x^{\prime}\right)\right)\right] \\
& \mathscr{H}\left(v_{2}, v_{3}\right)=4 \pi \tau\left(\varepsilon^{*}\right) \int d v_{3}^{\prime} v_{3}^{\prime 2} \\
& \times\left[\int d x G_{0}^{(4)}\left(v_{3}, \Pi_{6}\left(v_{2}, v_{3}^{\prime}, x\right), \Pi_{7}\left(v_{2}, v_{3}^{\prime}, x\right)\right) \mathscr{K}\left(\Pi_{6}\left(v_{2}, v_{3}^{\prime}, x\right), \Pi_{7}\left(v_{2}, v_{3}^{\prime}, x\right)\right)\right. \\
& \left.\quad+G_{0}^{(4)}\left(v_{3}, v_{2}, v_{3}^{\prime}\right) \mathscr{H}\left(v_{2}, v_{3}^{\prime}\right)\right]
\end{aligned}
$$


The coupled integral equations (5.3]) have been successfully solved for four-boson system and similar to three-boson case a scaling plot has been obtained which describe the universal tetramers

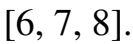

\section{Acknowledgments}

We acknowledge partial financial support from the Brazilian agencies Fundação de Amparo à Pesquisa do Estado de São Paulo and Conselho Nacional de Desenvolvimento Científico e Tecnológico.

\section{References}

[1] W. Glöckle, The quantum mechanical few-body problem (Springer, Berlin, 1983).

[2] A. Nogga, H. Kamada, and W. Glöckle, Phys. Rev. Lett. 85, 944 (2000).

[3] A. Nogga, H. Kamada, W. Glöckle, and B. R. Barrett, Phys. Rev. C 65, 054003 (2002).

[4] M. R. Hadizadeh and S. Bayegan, Few Body Syst. 40, 171 (2007).

[5] M. R. Hadizadeh, L. Tomio, and S. Bayegan, Phys. Rev. C 83, 054004 (2011).

[6] T. Frederico, L. Tomio, A. Delfino, M.R. Hadizadeh, and M.T. Yamashita, Few Body Syst. 51, 87 (2011).

[7] M. R. Hadizadeh, M. T. Yamashita, Lauro Tomio, A. Delfino, T. Frederico; Phys. Rev. Lett. 107 , 135304 (2011).

[8] M. R. Hadizadeh, M. T. Yamashita, Lauro Tomio, A. Delfino, T. Frederico; Phys. Rev. A 85, 023610 (2012). 